\title{
Recommendations for the Use of Online Social Support for African American Men
}

\author{
Daphne C. Watkins and S. Olivia Jefferson \\ University of Michigan
}

\begin{abstract}
African American men face greater psychosocial stressors than African American women and men of other racial and ethnic groups, which place them at higher risk for psychological distress. Yet, research suggests that African Americans are less likely to utilize professional mental health services because of their mistrust of the health care system and their need for more specialized and innovative services. Supplemental resources aimed at positive coping and social support for African American men may reduce the likelihood that they experience psychological distress, which could lead to more severe mental disorders. This article proposes the use of online social support for African American men who are in early, nonsevere stages of psychological distress. We examine the unique experiences of African American men, discuss distress among this underserved group, and finally, offer recommendations for achieving an online community for African American men.
\end{abstract}

Keywords: African American men, coping, online social support, psychological distress

Few studies explore informal coping strategies for African American men, yet this group tends to experience macro- and microaggressions derived from the historical context of discrimination and disadvantage, as well as issues that face the male gender (Bonhomme, 2004; Erguner-Tekinapl, 2009; Pieterse \& Carter, 2007). Of the studies that report coping mechanisms among African American samples, informal help-seeking strategies (or helpseeking that does not involve formally trained health professionals) are among the most frequently reported options used to address emotional concerns (Woodard et al., 2008; Woodard, Taylor, \& Chatters, 2011). Two decades of research suggests that African American men prefer to solve their problems on their own, or with the help of a friend or family member-not by using professional counseling services (Woodard et al., 2008, 2011). Given this, health professionals should consider supplemental resources (i.e., community-based resources, technology, media, and self-care) that could help foster relationships with and among African American men apart from the health care system.

This article examines online social support as a tool for African American men who desire informal, supplemental resources for managing and mediating their psychological distress. First, we explore the unique experiences of what it means to be both African American and male, and the subsequent challenges that African

This article was published Online First August 27, 2012

Daphne C. Watkins and S. Olivia Jefferson, School of Social Work, University of Michigan.

We acknowledge colleagues from the University of Michigan's Program for Research on Black Americans and the "Man up, Man down" project (Harold W. Neighbors, Wayne R. McCullough, Jamie Abelson, Deborah Coral, Brian Krenz, and Niki Matusko) for their support of this work.

Correspondence concerning this article should be addressed to Daphne C. Watkins, School of Social Work, University of Michigan, 1080 South University Avenue \#60, Ann Arbor, MI 48109-1106. E-mail: daphnew@ umich.edu
American men face because of their social conditions. Next, we examine distress for African American men, define social support and online social support, and then discuss these phenomena in the cultured and gendered context of African American men. Finally, we review current Internet-based programs for men and make recommendations for developing an online mental health "community" for African American men. Using the Internet to seek health information and obtain treatment by clinicians is beyond the scope of this article. Rather, we propose use of the Internet by African American men who have difficulty seeking face-to-face help during stressful life situations. This informal, supplemental resource could help to identify social support early in their mental health trajectories and, if needed, segue into more formal primary and secondary modes of care. With the increasing use of technology for knowledge acquisition and remote communication, the Internet serves as a resource for obtaining health information and services, as well as sharing feelings and thoughts related to health experiences (Dietrich, 2010; Dutta-Bergman, 2004).

\section{Scope of the Problem}

The social conditions and experiences of African American men differ from those of men from other racial and ethnic groups; thus, being an African American man has considerable implications for mental health. For example, fewer than $8 \%$ of African American men graduate from college compared to $17 \%$ of White men and $35 \%$ of Asian men (Kaiser Family Foundation, 2006). Beyond educational attainment, the unemployment rate for African American men is more than twice the rate of White, Hispanic, and Asian men. When compared to White, Hispanic, and Asian men of the same cohort, African American men between 16 and 29 years of age have less of a presence in the workforce (Kaiser Family Foundation, 2006). The inability to earn a living translates into $20 \%$ of African American men living in poverty, compared to $18 \%$ of Hispanic, $12 \%$ of Asian, and $10 \%$ of White men (U.S. Census 
Bureau, 2005). For many African American men, the abovementioned factors lead them toward "street" life, or a lifestyle that includes the network of public and semipublic social settings (e.g., street corners, bars, afterhours locations, drug houses, and vacant lots) that serve as important influences on their psychosocial development and life course trajectories (Oliver, 2006; Payne, 2011). Street life oftentimes subject African American men to the judicial system, where they represent more than $40 \%$ of the prison population, though only $14 \%$ of the total U.S. population (Harrison $\&$ Beck, 2005). Unless acted upon by protective factors, these psychosocial stressors result in poor mental health for African American men (Mizell, 1999; Watkins, Walker, \& Griffith, 2010).

Despite their increased exposure to various psychosocial stressors, there are mixed findings on the prevalence and severity of mental disorders for African American men. Results from large, epidemiologic community surveys imply that the prevalence of depression symptoms is lower in African American men compared to White men and African American women (Breslau et al., 2005). Other studies suggest that the rates of depression among African American men are not less than those of other men, but rather, that disorders tend to be underdiagnosed in men of color (Dallas \& Burton, 2004; Woodard et al., 2011). Still, some authors have suggested that regardless of whether or not prevalence rates are lower for Blacks, the course and persistence of mood disorders may be more chronic for Blacks than they are for Whites (Williams et al., 2007). Interpretations of these findings raise a couple of questions with regard to help seeking. First, can health and social services professionals do anything to intervene on the potentially deleterious mental health trajectory of some African American men? Second, given the lack of mental health service use by African American men, what strategies can we use to reach this population before they progress toward severe and persistent levels of depression, anxiety, and distress?

\section{Distress Defined}

Symptoms of distress include feeling downhearted or "blue," experiencing a lack of enthusiasm, having trouble falling or staying asleep, and feeling hopeless about the future. Previous studies have taken one of three overarching perspectives on the operationalization and measurement of distress in research and practice. In the first perspective, Mirowsky and Ross (2003) treat distress as an umbrella phenomenon and link it directly to mental health. According to this perspective, mental disorders are viewed as distinctively different and socially constructed phenomena (Mirowsky \& Ross, 2003). The second perspective acknowledges the presence (flourishing) and absence (languishing) of mental health and link the two on a continuum (Keyes, 2002). This so-called "mental health continuum" often includes well-being, defined as "the general sense of enjoying life and feeling happy, hopeful about the future, and as good as other people" (Mirowsky \& Ross, 2003, p. 26). Finally, the third perspective finds no underlying continuum across mental health, psychological distress, and mental illness and proposes, instead, a "discontinuous perspective" that views distress, illness, and disorder as distinctly and operationally different phenomena (Payton, 2009).

For the purposes of this article, we conceptualize distress using the framework proposed by Mirowsky and Ross (2003) because it has been used frequently as the underlying framework guiding studies on the mental health and well-being of African American men (Gaines, 2007; Lincoln, Taylor, Watkins, \& Chatters, 2011; Watkins, Green, Rivers, Rowell, 2006). Mirowsky and Ross (2003) define psychological distress as "the unpleasant subjective state of depression and anxiety, which have both emotional and physiological manifestations" (p. 8). These manifestations take on a second level of specificity, called mood and malaise. Mood refers to an individual's feelings (i.e., sadness, fear, loneliness) whereas malaise refers to an individual's physiological state (i.e., dizziness, headaches, and cold sweats).

\section{Distress Among African American Men}

For many African American men, the perils of distress begin at a very early age. Nearly one third of all African American youth live in poverty (Paxton, Robinson, Shah, \& Schoeny, 2004), which makes them particularly vulnerable to victimization and exposure to violence. Homicide is the leading cause of death for African American youth between the ages of 15 and 24, and increased exposure to violence has been linked to adverse psychological states among youth such as depression, anxiety, and suicidal ideation (Lindsey et al., 2006; Paxton et al., 2004). Feelings of loss, stress, and isolation associated with chronic exposure to violence influence symptoms of posttraumatic stress. Mazza and Reynolds (1999) found that posttraumatic stress symptoms are significantly related to exposures to violence, and that they mediate the relationship between exposure to violence and other forms of psychological distress, such as depression and suicidal ideation. Mazza and Reynolds also found that social support might have a buffering effect on psychological distress in their sample. Under certain circumstances, social support is protective against depression and other mental problems among African American adolescents (Paxton et al., 2004). Stressors that are germane to young African American men also have implications for their mental health over the life course.

African American men experience disproportionately higher levels of psychological distress due to their exposure to a greater frequency and severity of psychosocial stressors compared to other groups (Sellers, Bonham, Neighbors, \& Amell, 2009; Williams, 2003). The frequency and severity of psychosocial stressors are exacerbated by other sociodemographic factors (i.e., age, household income, marital status, education level) that can influence the emotional and psychological health of African American men. For example, the largest epidemiologic study of African Americans in the United States found that African American men aged 34 and younger experienced psychological distress at higher rates than those over the age of 35 (Lincoln, Taylor, Chae, \& Chatters, 2010; Lincoln et al., 2011; Watkins, Hudson, Caldwell, Siefert, \& Jackson, 2011). Likewise, studies have chronicled the psychological distress of African American men as a result of discrimination (Pierre \& Mahalik, 2005; Pieterse \& Carter, 2007; Sellers et al., 2009), negative attitudes toward the criminal justice system (Gaines, 2007), racial and cultural identity (Pierre \& Mahalik, 2005; Wester, Vogel, Mei, \& McLain, 2006), depression (Lincoln et al., 2011; Watkins et al., 2011), violence (Paxton et al., 2004), and issues surrounding masculine gender norms (Pierre, Mahalik, \& Woodland, 2001). Psychosocial coping and socioeconomic challenges faced by African American men have a negative impact on their mental health (Lincoln et al., 2011; Watkins et al., 2006; 
Watkins et al., 2010). Psychological distress among African American men is a problem because of its silent presence in the African American community. The term "silent" is used here because although communities witness the direct and indirect effects of distressed African American men, this group is undiagnosed, underdiagnosed, untreated and undertreated for their mental health problems (Woodard et al., 2011).

\section{Underutilization of Mental Health Services by African American Men}

Reports have demonstrated greater dissatisfaction with the health care system, less access to quality mental health care, and greater unmet mental health care needs among Blacks compared to Whites (Smedley, Stith, \& Nelson, 2002; Wells, Klap, Koike, \& Sherbourne, 2001). Three decades of research have suggested that African Americans may refuse to utilize mental health services due to their mistrust of the health care system or their needs and concerns that require specialized and innovative services in which mental health care systems may not be able to provide. Researchers in the early 1980s portrayed Blacks as passive, externally motivated by situations, and lacking in the characteristics needed to control and direct their own destinies (Chunn, Dunston, \& Ross-Sheriff, 1983). Yet studies that are more recent have uncovered other characteristics, such as the presence of stoicism and self-concealment in the African American community, that result in fewer African American men seeking formal and informal social support (Baker, 2001; Watkins et al., 2010).

From an individualistic perspective, several authors have explained why African American men are less likely to seek formal mental health services (Whaley, 2001). For example, Woodward, Taylor, and Chatters (2011) suggested that African American men do not voluntarily seek mental health services because they are socialized to handle difficulties or problems by not demonstrating stress or suppressing the intensity of stressors. Majors, Tyler, Peden, and Hall (1994) offered the "cool pose" phenomenon in which African American men behave in a certain, nonchalant manner in order to cope with discrimination, invisibility, frustration, and education and employment barriers. From a community perspective, members of underserved racial and ethnic groups are less likely to seek formal mental health services because they experience poor treatment due to the negative attitudes (i.e., biases, stereotypes) associated with their group (Karlsen, 2007). This problem is intensified among African American men, who are less likely to recognize that they are experiencing mental health problems, and more reluctant to seek help due to a fear of jeopardizing their masculine ideologies (Addis \& Mahalik, 2003; Brooks, 2001; Mahalik, Good, \& Englar-Carlson, 2003; Rochlen \& Hoyer, 2005) and/or racial identities (Parham, White, \& Ajamu, 2000). African American men may also avoid seeking mental services due to the stigma associated with formal services and a belief that they will experience discrimination (Keating \& Robertson, 2004).

Lastly, from a systems perspective, navigating the health care system and the challenges therein (e.g., quality of health services, mental health services, and correctional health services provided to African American men) presents an additional barrier, met with further opposition when almost 4 out of every 10 African American men lack health insurance (Kaiser Family Foundation, 2006). There is a need for supplemental resources that do not aim to take the place of formal care, but rather provide a supportive network for African American men (a) who may be less likely to disclose their mental status face-to-face, (b) whose distress may be less severe, and (c) who may need a gradual progression toward more formal care through a safe and virtually anonymous forum. With societal pressures of fulfilling gender and cultural norms (Wester et al., 2006), African American men would benefit from having exposure to and engaging in a nonthreatening community where they can discuss their distress and its appending symptoms.

\section{Social Support}

Social support is a reciprocal process involving social networks of close relationships (intimates, family, and friends) and weak ties (coworkers, acquaintances, and strangers) (Albrecht \& Goldsmith, 2003). Social support affects health by regulating thoughts, feelings, and behaviors to promote health; by fostering an individual's sense of meaning in life; and by facilitating health-promoting behaviors (Wang, Wu, \& Liu, 2003). The lack of social support may adversely affect health, so social support is an integral component of the health and well-being of the individual as a whole. As one of the most important domains of human relationships, the capacity to establish and sustain friendships is a critical indicator of healthy psychological development (Fehr, 2004). In fact, the level of support people receive from their friends has been linked to a variety of outcomes such as improved physical and mental health, coping efficacy, and improved self-esteem (Fehr, 2004). In social science literature, social support is defined in various ways. Two of the most common ways are enacted support (received) and perceived support (anticipated).

Enacted support is the emotional, informational, and instrumental support offered by significant others. Perceived support is the individual's subjective sense of satisfaction with support and his or her ability to anticipate support from others (Rhodes \& Lakey, 1999). Generally, among African Americans, friends play a large role in addressing emotional concerns. Thus, enacted support is a primary objective in many male and female relationships. The degree to which perceived support is achieved may vary by gender. For example, Franklin (1992) reported that for African American men, an ability to talk about everything in their lives with their friends leads to friendships that are warmer and more intimate. Franklin also found that although gender socialization may influence men to withhold some forms of sharing, the African American men who did experience affective and informational exchanges in their friendships also perceived those friendships as exceedingly important. Not only do enacted and perceived support vary by gender, but so do age differences in friendships among African American men. Mattis et al. (2001) found that participants 35 years of age and older scored significantly lower on advice sharing and emotional sharing with friends of either gender when compared to younger men. Furthermore, the influence of religion may also play a role in the ability for African Americans to receive social support (Mattis et al., 2001), as it has been documented that religious involvement is negatively associated with psychological distress among this population (Lincoln \& Chatters, 2003).

Social support influences an individual's psychological wellbeing in a number of ways. Gathering resources for social support can provide individuals with encouragement and hope, feedback on one's health behavior and risks, a model of healthy behavior for 
health promotion, information about health, help with coping strategies, and lay referrals to health professionals (Albrecht \& Goldsmith, 2003). Due to the reciprocity that occurs during social support, its positive components make it vital in mental health education. Findings from a meta-analysis indicated that individuals who obtained higher levels of social support might have a more positive health status, role function and behaviors, psychosocial adjustment, adjustment to life, coping behaviors, health beliefs, health promotion behaviors, quality of life, well-being, and selfactualization (Wang et al., 2003). With the technological advances that have emerged in the new millennium, social support may be delivered face-to-face, over the telephone, and over the Internet, also known as "online social support."

\section{Online Social Support}

Online social support defined. The theory of online social support (OSS) was proposed by LaCoursiere (2001) to address gaps in nursing's theoretical models that consider issues related to the social environment. OSS theory assists in the holistic conceptualization of online social support, linking online phenomena with traditional quantitative and qualitative methods of description. It also provides an integrated basis for further theory development and related research. Its purpose is not to describe health information-seeking behavior that occurs on the Internet, such as through a search engine, but rather the process of support that "is characterized by interpersonal relationships with other unseen persons who are mostly unknown to each other" (LaCoursiere, 2001, p. 65). During this process, information exchanged and interactions between individuals are the chief phenomenon.

Online social support groups are virtual communities. They share similar goals as face-to-face support groups; however, online groups provide a certain amount of anonymity that one may not experience in a face-to-face support group. Anonymity allows for the discussion of potentially embarrassing topics or otherwise taboo subjects, increases the potential of self-disclosure, and encourages honesty and intimacy among the participants when discussing potentially stigmatizing topics, such as those related to mental health (Mulveen \& Hepworth, 2006; White \& Dorman, 2001). Compared to face-to-face groups, online support groups also offer greater access, are more convenient, are less spontaneous, have few (if any) nonverbal responses, and rely heavily on written text (Kernsmith \& Kernsmith, 2008; LaCoursiere, 2001). The various types of online groups include web-based message boards, newsgroups, videoconference meetings, listservs, "chat" sessions, and discussion forums (Dietrich, 2010).

Internet support groups are self-help in nature, rather than therapeutic. Studies have reported both positive and negative outcomes from Internet usage, most notably, that it either increases or decreases depression over time (Morgan \& Cotton, 2003; LaRose, Eastin, \& Gregg, 2001; Wright, 2000). For example, using a college student sample, LaRose and colleagues found that frequent email exchanges increased perceived social support, which in turn alleviated depression. On the contrary, Morgan and Cotton (2003) found that depression symptoms increased when their college sample engaged in email and chat room/instant-messaging use. For older adults, Wright (2000) found that level of participation in online social support groups decreased participants' perceived stress and increased their potential to create strong relationships online.

African American men as online social support participants. The functioning of an African American household encompasses a large network of kinship and family support beyond biological relatives, such as uncles, aunts, clergy, and significant others. In terms of social support, studies have found that compared to Whites, high levels of critical and intrusive behaviors by family members predicted better mental health outcomes for African Americans (Rosenfarb, Bellack, \& Aziz, 2006). The authors suggested that the cultural beliefs of African American families consider "confrontation" as an expression of concern. Unfortunately, the topic of social support among African American men is grossly understudied. Among the exceptions are studies that underscore the relationships of socially marginalized men, such as those deep-rooted in gang activity or life on "the streets" (Oliver, 2006; Payne, 2011), the criminal justice system (Gaines, 2007), homeless men (Littrell \& Beck, 2001), and low-income nonresidential fathers (Anderson, Kohler, \& Letiecq, 2005).

Despite the insufficient research on social support networks among African American men, other studies suggest that kinship and social support help African American men maintain a sense of community for their health (Parham et al., 2000; Plowden \& Young, 2003). An example of this is the influence of significant others as a critical factor in motivating urban African American men to seek health care and participate in health-related activities. Similarly, the influence of trusted and respected individuals (or family members, political officials, and members of the media) have also been acknowledged among African American male samples (Plowden, John, Vasquez, \& Kimani, 2006). Race was absent during the discussion about preferred outreach methods for African American men. Instead, participants discussed the importance of communicating with men with whom they shared common characteristics — such as economic status and age-as individuals whom they considered helpful in connecting them with their health needs. Among African American men, social support is of high importance; however, issues of privacy and anonymity may emerge when African American men seek informal or formal help (Woodward et al., 2011).

The stigma associated with individuals who may have mental health problems can also discourage African American men from seeking professional help. Therefore, the anonymity that exists with online social support will be beneficial for African American men who want to participate in an online community. The use of the online social support groups has quadrupled in the past decade (LaCoursiere, 2001; Lieberman \& Goldstein, 2005; Mulveen \& Hepworth, 2006; Sullivan, 2003). Therefore, the ability to access the online community at any time of the day or night without other participants knowing the user's identity may provide a safe haven, particularly for men (Pinnock \& Jones, 2003). In addition, when users know that other users are unaware of their identity, they may feel more comfortable disclosing personal information. As a result, discussing topics that would otherwise make face-to-face social groups uncomfortable can be easily facilitated in an online support group such as stress associated with work, racism, and sexism to name a few (Albrecht \& Goldsmith, 2003; Chang \& Yeh, 2003; Mulveen \& Hepworth, 2006). The online group format is also a little less spontaneous than face-to-face encounters, thereby providing participants the opportunity to think about their responses 
before they share them with the rest of the group (Kernsmith \& Kernsmith, 2008). Gender identities, as it pertains to how African American men define manhood, are equally important. Online users can be more sensitive of the problems experienced by other African American men, a gender identity that may not be well received during face-to-face meetings. In addition, African American men can support each other as they express their thoughts and feelings about their emotional and psychological health.

\section{The Findings: Internet-Based Programs for Men}

Men have not benefited from advances in the use of the Internet for social support to groups with unique backgrounds and sensitive needs (Dietrich, 2010; Wasserman \& Richmond-Abbott, 2005). Examples of unique backgrounds include men from economically disadvantaged communities and sensitive needs include men with terminal illness, emotional challenges, and personality disorders that prevent them from thriving in face-to-face interactions with other people. We conducted a very specific, yet thorough search of the literature for Internet-based programs specific to African American/Black men and informal mental health social support. We searched computer databases (e.g., Medline, PsycINFO, Pub Med, EBSCOhost, and Science Direct) for peer-reviewed article using keywords such as African American men, Black men, Internet, Web-based, programs, support, groups, networking, telehealth, telemental health, interventions, mental health, stress, distress, and psychological distress. Our search did not uncover any articles about using the Internet as a means of social support for African American men. Noting this, we decided to conduct a broader search for articles that discussed using online social support for men's health. We did not limit the search to just "African American" and "Black men," and similarly, we broadened the search to include not just mental health but all health conditions and health-related problems. Doing this uncovered a total of eight articles, two that explored using the Internet as a means of delivering online social support for health conditions among men and an additional six about online education for men. This broader search excluded studies that discussed specific subgroups of men using the Internet for means other than seeking social support (e.g., homosexual men's use of the Internet to seek partners or men with HIV/AIDS and their use of the Internet for health information). Below, we briefly examine the findings and suggestions uncovered from the literature for the purposes of developing an online social support group for men.

\section{Online Social Support of Men}

Few studies have highlighted the unique experiences of men in online social support settings. Though we could not locate a report of an online social support group for African American men with psychological distress, we did find a report that tested the idea of using online social support groups for Asian American men to help them combat racial, cultural, gender, and treatment issues (Chang $\&$ Yeh, 2003). The authors discussed the importance of differentiating between open online groups, which can be accessible by the general public, and closed online groups, which are limited to a selected group of individuals. Petersen's (2009) thematic analysis of online social support groups for men living with HIV suggested that not the mere availability of online support, but rather, the quality of support is vital, particularly with stigmatized illness. Petersen suggested linking support groups and people with support needs through common communicative preferences (e.g., positivity) and actions that helped maintain group cohesion was an effective strategy for members of an online group for stigmatized illnesses. A "speech community" was developed and "included norm-enforcing behaviors, such as the use of a moderator, the overt discussion of group rules, and the use of introductions as a group-building device" (p. 536). Also noteworthy was the support seeking behaviors observed, as it was often indirect, but when it was direct, it involved asking others to share their personal experiences.

\section{Online Education for Men}

Our review of the literature uncovered six studies that discussed online education for men. Though online education was beyond the scope of this article, we want to highlight some noteworthy aspects of this area of study. First, prostate cancer was the primary illness discussed in this literature. Two studies discussed using the Internet for prostate cancer education among African American men and one study reported on the prostate cancer information needs of Australian men (Pinnock \& Jones, 2003; Wallington, 2008; Weston et al., 2007). Of the three remaining studies that discussed online education for men, one of them tested the feasibility of a telehealth psycho-educational intervention for persons with schizophrenia and their families (Rotondi et al., 2005). The other two evaluated computer assisted programs for men-one focused on depressive symptoms of persons living with HIV/AIDS (Lai, Larson, Rickoff, \& Bakken, 2008) and the other targeted weight-loss in overweight and obese men (Morgan, Lubans, Collins, Warrne, \& Callister, 2009).

\section{Achieving the Online "Community" for African American Men}

The isolated approach that some African American men take to problem solving is a barrier to their mental health and well-being. Therefore, an online community of social support may be a useful medium for mental health promotion for this group. An online community targeted toward African American men could empower them to take control of their mental health and assist them in feeling more comfortable about discussing their mental conditions with others - it could even lead to consultation with a mental health professional, if necessary. However, achieving the online community for African American men involves assessing the needs of the group as well as planning, implementing, and evaluating the effectiveness of the online community for African American men. Below, we propose four recommendations.

\section{Recommendation 1: Use Theoretical Frameworks Derived From Previous Literature on African American Men}

Theory is essential to the success of programs. Therefore, health and social service professionals are encouraged to use conceptual frameworks to guide the steps toward developing an online community for African American men. Chang and Yeh (2003) pro- 
vided practical guidelines based on theoretical frameworks derived from group therapy and racial identity in their study of online social support groups for Asian American men. They also suggested that their processes would apply to facilitated online social support groups for men of other racial and ethnic backgrounds. Online social support communities grounded in gender- and racebased theoretical frameworks will help move us toward more culturally sensitive and gender-specific programming for African American men. The literature has highlighted three primary conceptual perspectives to consider for work with African American men: historical and social adaptation; lack of privilege and power; and Black male identity and gender role socialization.

The historical and social adaptation perspective suggests that the impact of racial discrimination is detrimental to the mental health of Black men and must be approached with careful consideration for the obvious and underlying factors involved. The historical impact of racism in the context of migration, alienation, and the way in which African American men are stigmatized in society is important to recognize in developing support groups. Pierre, Mahalik, and Woodland (2001) describe a historical and social adaptation framework for understanding issues of masculinity among African American men, a social determinant that has received growing attention in the gender and mental health literature (Watkins, 2011). The historical and social adaptation perspective suggests that African American men are likely to have insight into the reality of their situations, but are not systematically included in the conversation associated with how to ameliorate mental health concerns. For instance, African American men have not historically been considered active stakeholders in their mental health and well-being. However, they tend to value health education and health maintenance; and therefore, may be receptive to preventive and health promotion strategies (Ravenell, Johnson, \& Whitaker, 2006; Watkins, 2011; Watkins et al., 2006).

The lack of privilege and power perspective posits that what African American men have had to endure with respect to their social class and economic status has an influence on their masculine ideology and mental health. Feminist paradigms are examples of the types of perspectives that have been used to cross-fit traditional disciplinary boundaries of gender and gender-related studies. Feminist paradigms with respect to masculinity and mental health illustrate "the degree to which power differences between men and women are seen as central to any analysis of gender" (Addis \& Cohane, 2005, p. 642). From a feminist perspective, gender is a multilevel system in which men cannot be understood beyond the privileged positions that they hold in society. Consequently, African American men find themselves in impoverished settings, where few economic opportunities exist because of social inequalities and racism (Utsey, 1997). Payne (2006) argued that, for some African American men, "street-life," which includes illegal behavior, is adaptive in the absence of academic and economic resources; primarily because they are an economically and socially marginalized group and do not have access to the White male power and economic structure. Because mental health outcomes are largely impacted by resource, opportunity, and environmental differences by race (Kessler et al., 1994; Williams \& Williams-Morris, 2000), over time the lack of privilege and power can generate rage, anger, frustration, resentment, grief, and despair for African American men.
Finally, the Black male identity and gender role socialization perspective defines men's sense of identity by evaluations of how well they feel they are accomplishing social roles that are important to them (Bowman, 1989). Black men have often struggled to achieve success in highly valued social roles and appropriate age-related accomplishments such as being a good provider, spouse/ partner, father, or professional/ employee. Therefore, success in any one of these areas is a challenging priority that supersedes emotional and physical health (Bowman, 1989; Courtenay, 2000). Even when middle-class Black men earn incomes similar to those of their White counterparts, doing so does not translate to more desirable housing and neighborhood conditions, economic stability, equivalent levels of wealth, or income levels that reflect level of education (Braboy-Jackson \& Williams, 2006; Williams, 2003). Poor mental health can result from maladaptive coping strategies (e.g., substance abuse) implemented to resolve the discrepancy between desire to provide and ability to do so (Jackson \& Knight, 2006). Among marginalized men, masculine power is displayed in unhealthy forms because of the inaccessibility of professional and economic achievements (Courtenay, 2000). Directly and indirectly, the stressors associated with male identity and male gender socialization can lead to limited economic opportunities and social marginalization. Furthermore, as African American adults tend to conceal emotional health status (Baker, 2001), researchers have reported positive associations between self-concealment and indications of compromised mental health, such as depression, poor self-esteem, and lower levels of perceived social support (Cramer \& Barry, 1999). It has also been suggested that African American men have poor health because of their likelihood of adhering to traditional masculine norms that promote avoidance of medical care and tendency to exhibit traditional masculine norms that are detrimental to their health (Royster, Richmond, Eng, \& Lewis, 2006; Watkins, Green, Goodson, Guidry, \& Stanley, 2007; Watkins \& Neighbors, 2007).

\section{Recommendation 2: Identify Potential Barriers and Facilitators for African American Men's Use of an Online Community}

A number of psychosocial factors may prevent African American men from participating in an online community. First, because of the expenses associated with Internet connectivity (Dietrich, 2010), low-income men may not have access to a computer, or much less a computer that is Internet capable. However, the so-called "digital divide" has reportedly closed as more African Americans are steadily gaining access to and ease with the Internet (Marriott, 2006). In fact, $45 \%$ of online African Americans say the Internet helps them get health information compared to $35 \%$ of Whites (Pew Online Life Report, 2000). Second, a public (i.e., library, community center, church) or private (i.e., home, office) setting can also influence the likelihood of a man initiating and maintaining his presence in the online community. With the growing popularity of cellular phones, PDAs, smartphones, and tablets, accessing the Internet from various locations beyond home and work has become a social norm. Next, after an African American man acquires access to the Internet and engages in the online community, there may be a learning curve with regard to his computer knowledge, language, typing skills, and experience, which could affect his presence in the online community. Although 
some studies have reported age as a determining factor for Internet comfort and use, others have reported positive outcomes from using the Internet for social support regardless of age (Dietrich, 2010; LaRose et al., 2001; Wright, 2000). Thereby, when developing an online community for African American men, emphasis should fall on the age, skill level, and comfort of the participants. The importance of these factors can be illustrated through the underrepresentation of African American men and women in online social support groups for cancer due to culture-specific support preferences (Fogel, Ribisl, Morgan, Humphreys, \& Lyons, 2008). For example, African Americans tend to use more religious resources of support-as this is more culturally congruent for African Americans than Internet-based support (Fogel et al., 2008). As African Americans age and obtain support from kin networks, fellow church members and adult children, they are more likely to seek more face-to-face intergenerational support for emotional and life crisis problems than Whites. In addition, preference with regard to written versus oral expression has be noted among Whites compared to African Americans (Banks-Wallace, 2002).

Finally, we would be remiss to exclude the potential challenges that evolve from how the desire for seeking help can influence what it means to be a man, overall, and apart from Internet-based resources. In addition, the influence of social stigma on men may lead to lambasting from family and friends for simply expressing a desire to seek help or mental health care. Therefore, it is important to recognize the online community as a safe space for men to share and receive the necessary support. Furthermore, the psychological effects of social norms and one's conformity to masculine ideologies influence a man's behavior whether he is alone or with others (Addis \& Mahalik, 2003).

\section{Recommendation 3: Consider the Use of "Tailored" Messages and Social Media}

When designing an Internet-based program for African American men, mental health professionals should consider that more localized information in an online community proves to be more effective than global information (Pinnock \& Jones, 2003). Due to the vast array of information and resources available on the Internet, it is important to include content that is more central to the participants' specific geographic region, educational level, and interests. For example, information that is germane to older African American men in southern U.S. states with an eighth grade reading level may not be appropriate for middle-aged African American men from midwestern U.S. states with graduate or professional degrees. Important components of targeted message development include ensuring that individuals' needs are assessed, that health professionals work with individuals to develop effective messages, and that health professionals meet participants "where they are" (Krueter, Lukwago, Bucholtz, Clark, \& SandersThompson, 2003).

Health and social service professionals should be sure to explore several ways to use the anonymity and confidentiality of the Internet to serve their male clientele. Similarly, developing creative strategies would not only engage men at the onset of a program, but also retain men over time. Such creative strategies could model many of the characteristics of current social media forums such as Facebook, MySpace, and Twitter. Examples in- clude e-newsletters where the success stories of distressed men are highlighted, online social support forums that allow men to exchange approved media that helped them cope (i.e., poems, stories, photos), and chat rooms and blogs where mental health professionals can respond to the men's inquiries. The ability to access these resources via smart phones is another way of making them accessible to participants 24 hours a day. These efforts could be essential to maintaining positive mental health during stressful times and provide access to more formal help-seeking options, should the need arise.

\section{Recommendation 4: Acknowledge the Roles of Health and Social Service Professionals Who Have Interest in and Experience With African American Men's Mental Health}

Generally, research and programming that address the mental health needs of African American men is lacking. Stereotyping and preconceived notions of African American men by mental health professions (Scott \& Davis, 2006) also hinders the success of therapeutic services as well as creative strategies for reaching this group. Health and social service professionals should strive to gain an understanding of African American men in the context of their daily life experiences and tailor health and well-being efforts to such experiences (Watkins et al., 2010). Health and social service professionals can also play a role in increasing the number of African American men who would consider participating in an online social support community by empowering them to take control of their health and the utilization of online social support. The overarching goal of examining attitudes, subjective norms, and perceived behavior control as it relates to African American men is to encourage the behavioral intention to actively participate in the online community, feel a sense of ownership within that community, and then achieve improved mental health. It is essential to understand African American men and their families in the midst of the intrapersonal and environmental adversities that affect them and predispose them to distress, because this oftentimes limits their ability to make use of health resources. In addition, it is important to articulate how we can move health and social service professionals toward a deeper understanding of the culturally sensitive and gender-specific phenomena that contribute to the complexity of mental health among African American men (Watkins et al., 2010). Previous studies show that African American men who have positive exchanges with other African American men tend to view these relationships as exceedingly important (Elligan \& Utsey, 1999; Franklin, 1997). African American men who are engaged in an online community to help mediate their distress could satisfy their strong desire of social support and alleviate their longing for a voice in the mental health community.

\section{Conclusion}

The purpose of this article was to examine the use of online social support for African American men who desire an informal medium for discussing their psychological health. Due to the culture- and gender-specific stigma surrounding men who seek professional help for mental health issues, African American men may be more inclined to seek professional help after using informal resources that act as stepping-stones toward engagement in 
more formal help-seeking behaviors (Marriott, 2006). Online support may be an effective approach for some African American men, such as those who are less likely to disclose their mental status face-to-face, those who experience less severe distress, and/or those who need a gradual progression toward more formal care through a safe and virtually anonymous online forum. African American men are at risk for numerous social conditions and health risks. Developing interventions that protect African American men from exposure to traumatic experiences may be counterproductive toward reducing the likelihood that they experience psychological distress. Instead, efforts should include online social support as a preliminary step toward helping African American men maintain mental health and well-being. African American men may be more inclined to seek help for their psychological distress if they first explore their emotions through a medium with which they feel comfortable, such as the Internet.

\section{References}

Addis, M. E., \& Cohane, G. H. (2005). Social scientific paradigms of masculinity and their implications for research and practice in men's mental health. Journal of Clinical Psychology, 61, 633-647. doi: 10.1002/jclp.20099

Addis, M. E., \& Mahalik, J. R. (2003). Men, masculinity, and the context of help seeking. American Psychologist, 58, 5-14. doi:10.1037/0003066X.58.1.5

Albrecht, T. L., \& Goldsmith, D. J. (2003). Social support, social networks, and health. In T. L. Thompson, A. Dorsey, R. Parrott, and K. Miller (Eds.), The handbook of health communication (pp. 263-284). Hillsdale, NJ: Erlbaum.

Anderson, E. A., J. K. Kohler, \& Letiecq, B. L. (2005). Predictors of depression among low-income, nonresidential fathers. Journal of Family Issues, 26, 547-567. doi:10.1177/0192513X04272753

Baker, F. M. (2001). Diagnosing depression in African Americans. Community Mental Health Journal, 37, 31-38. doi:10.1023/A: 1026540321366

Banks-Wallace, J. (2002). Talk that talk: Storytelling and analysis rooted in the African American oral tradition. Qualitative Health Research, 12, 410-426. doi:10.1177/104973202129119892

Bonhomme, J. J. E. (2004). The health status of African-American men: Improving our understanding of men's health challenges. Journal of Men's Health \& Gender, 1, 142-146. doi:10.1016/j.jmhg.2004.07.004

Bowman, P. J. (1989). Research perspectives on Black men: Role strain and adaptation across the adult life cycle. In R. L. Jones (Ed.), Black adult development and aging (pp. 117-150). Berkeley, CA: Cobb \& Henry.

Braboy-Jackson, P., \& Williams, D. (2006). Culture, race/ethnicity, and depression. In C. L. M. Keyes \& S. H. Goodman (Eds.), Women and depression: A handbook for the social, behavioral, and biomedical sciences (pp. 328-359). New York, NY: Cambridge University Press.

Breslau, J., Kendler, K., Su, M., Gaxiola-Aguilar, S., \& Kessler, R. (2005). Lifetime risk and persistence of psychiatric disorders across ethnic groups in the United States. Psychological Medicine, 35, 317-327. doi:10.1017/S0033291704003514

Brooks, G. R. (2001). Masculinity and men's mental health. Journal of American College Health, 49, 285. doi:10.1080/07448480109596315

Chang, T., \& Yeh, C. J. (2003). Using online groups to provide support to Asian American men: Racial, cultural, gender, and treatment issues. Professional Psychology: Research and Practice, 34, 634-643. doi: 10.1037/0735-7028.34.6.634

Chunn, J. C., Dunston, P., \& Ross-Sheriff, F. (Eds.). (1983). Mental health and people of color. Washington, DC: Howard University Press.

Courtenay, W. H. (2000). Constructions of masculinity and their influence on men's well-being: A theory of gender and health. Social Science \& Medicine, 50, 1385-1401. doi:10.1016/S0277-9536(99)00390-1

Cramer, K. M., \& Barry, J. E. (1999). Conceptualizations and measures of loneliness: A comparison of subscales. Personality and Individual Differences, 27, 491-502. doi:10.1016/S0191-8869(98)00257-8

Dallas, C., \& Burton, L. (2004). Health disparities among men from racial and ethnic minority populations. Annual Review of Nursing Research, 22, 77-100.

Dietrich, C. (2010). Online social support: An effective means of mediating stress. Student Pulse Academic Journal, 2, 1-10. Retrieved from http:// www.studentpulse.com/a?id=173

Dutta-Bergman, M. J. (2004). Primary sources of health information: Comparisons in the domain of health attitudes, health cognitions, and health behaviors. Health Communication, 16, 273-288. doi:10.1207/ S15327027HC1603_1

Elligan, D., \& Utsey, S. O. (1999). Utility of an African centered support group for African American men confronting societal racism and oppression. Cultural Diversity and Ethnic Minority Psychology, 5, 156-165. doi: 10.1037/1099-9809.5.2.156

Erguner-Tekinapl, B. (2009). Daily experiences of racism and forgiving historical offenses: An African American experience. International Journal of Human and Social Sciences, 4, 1-9.

Fehr, B. (2004). Intimacy expectations in same-sex friendships: A prototypical interaction pattern model. Journal of Personality and Social Psychology, 86, 265-284. doi:10.1037/0022-3514.86.2.265

Franklin, C. W. (1992). "Hey, home-Yo, bro." In P. Nardi (Ed.), Men's friendships (pp. 201-214). Thousand Oaks, CA: Sage.

Fogel, J., Ribisl, K. M., Morgan, P. D., Humphreys, K., \& Lyons, E. J. (2008). The underrepresentation of African Americans in online cancer support groups. Journal of the National Medical Association, 100, 705-712.

Gaines, J. S. (2007). Social correlates of psychological distress among adult African American males. Journal of Black Studies, 37, 827-858. doi: $10.1177 / 0021934705285566$

Harrison, P. M., \& Beck, A. J. (2005). Prison and jail inmates at midyear 2005. United States Department of Justice, Bureau of Statistics. Retrieved from http://www.bjs.gov/content/pub/pdf/pjim05.pdf

Jackson, J. S., \& Knight, K. M. (2006). Race and self-regulatory health behavior: The role of the stress response and the HPA axis in physical and mental health disparities. In L. L. Carstensten \& K. W. Shaie (Eds.), National datasets and Black Americans: Social structure, aging and self-regulation in the elderly (pp. 189-240). New York, NY: Springer.

Kaiser Family Foundation (KFF). (2006). Race, ethnicity, \& health care fact sheet. Young African American men in the United States. Retrieved from:www.kff.org

Karlsen, S. (2007). Ethnic inequalities in health: The impact of racism. Better Health Briefing, 3. London, England: Race Equality Foundation. Retrieved from http://www.better-health.org.uk/

Keating, F., \& Robertson, D. (2004). Fear, Black people and mental illness: A vicious circle? Health and Social Care in the Community, 12, 439447. doi:10.1111/j.1365-2524.2004.00506.x

Kernsmith, P., \& Kernsmith, R. (2008). A safe place for predators: Online treatment of recovering sex offenders. Journal of Technology in Human Services, 26, 223-238. doi:10.1080/15228830802096598

Kessler, R. C., McGonagle, K. A., Zhao, S., Nelson, C. B., Hughes, M., Eshleman, S., . . . Kendler, K. S. (1994). Lifetime and 12-month prevalence of DSM-III-R psychiatric disorders in the United States: Results from the national comorbidity survey. Archive of General Psychiatry, 51, 8-19.

Keyes, C. L. M. (2002). The mental health continuum: From languishing to flourishing in life. Journal of Health and Social Research, 43, 121-140.

Krueter, M. W., Lukwago, S. N., Bucholtz, D. C., Clark, E. M., \& Sanders-Thompson, V. (2003). Achieving cultural appropriateness in 
health promotion programs: Targeted and tailored approaches. Health Education and Behavior, 30, 133-146. doi:10.1177/1090198102251021

LaCoursiere, S. P. (2001). A theory of online social support. Advances in Nursing Science, 24, 60-77.

Lai, T-Y., Larson, E. L., Rickoff, M. L., \& Bakken, S. (2008). User acceptance of HIV TIDES-Tailored interventions for management of depressive symptoms in persons living with HIV/AIDS. Journal of the American Medical Informatics Association, 15, 217-226. doi:10.1197/ jamia.M2481

LaRose, R., Eastin, M. S., \& Gregg, J. (2001). Reformulating the internet paradox: Social cognitive explanations of Internet use and depression. Journal of Online Behavior, 1. Retrieved from http://www.behavior.net/ JOB/v1n2/paradox.html

Lieberman, M. A., \& Goldstein, B. A. (2005). Self-help on-line: An outcome evaluation of breast cancer bulletin boards. Journal of Health Psychology, 10, 855-862. doi:10.1177/1359105305057319

Lincoln, K. D., \& Chatters, L. M. (2003). Keeping the faith: Religion, stress, and psychological well-being among African American women. In D. R. Brown \& V. M. Keith (Eds.), In and out of our right minds: African American women and mental health (pp. 233-241). New York, NY: Columbia University Press.

Lincoln, K. D., Taylor, R. J., Chae, D. T., \& Chatters, L. M. (2010). Demographic correlates of psychological well-being and distress among older African Americans and Caribbean Black adults. Best Practices in Mental Health: An International Journal, 6, 103-126.

Lincoln, K. D., Taylor, R. J., Watkins, D. C., \& Chatters, L. (2011). Correlates of psychological distress and major depressive disorder among African American men. Research on Social Work Practice, 21, 278-288. doi: 10.1177/1049731510386122

Lindsey, M. A., Korr, W. S., Broitman, M., Bone, L., Green, A., \& Leaf, P. J. (2006). Help-seeking behaviors and depression among African American adolescent boys. Social Work, 51, 49-58.

Littrell, J., \& Beck, E. (2001). Predictors of depression in a sample of African American homeless men: Identifying effective coping strategies given varying levels of daily stressors. Community Mental Health Journal, 37, 15-29. doi:10.1023/A:1026588204527

Mahalik, J. R., Good, G. E., \& Englar-Carlson, M. (2003). Masculinity scripts, presenting concerns, and help seeking: Implications for practice and training. Professional Psychology: Research and Practice, 34, 123-131. doi: 10.1037/0735-7028.34.2.123

Majors, R., Tyler, R., Peden, B., \& Hall, R. (1994). Cool pose: A symbolic mechanism for masculine role enactment and coping by Black males. In R. G. Majors \& J. U. Gordon (Eds.), The American Black male (pp. 245-260). Chicago, IL: Nelson-Hall.

Marriott, M. (2006, March 31). Digital divide closing as blacks turn to Internet. New York Times. Retrieved from http://www.nytimes.com/ 2006/03/31/us/31divide.html?

Mattis, J. C., Murray, Y. E., Hatcher, C. A., Hearn, K. D., Lawhon, G. D., Murphy, E. J., \& Washington, T. A. (2001). Religiosity, spirituality, and the subjective quality of African American men's friendships: An exploratory study. Journal of Adult Development, 8, 221-230. doi: 10.1023/A:1011338511989

Mazza, J. J., \& Reynolds, W. M. (1999). Exposure to violence in young inner-city adolescent: Relationships of suicidal ideation, depression, and PTSD symptomatology. Journal of Abnormal Child Psychology, 27, 203213. doi:10.1023/A: 102190042300

Mirowsky, J., \& Ross, C. E. (2003). Education, social status, and health. Hawthorne, NY: Walter de Gruyter, Inc.

Mizell, A. C. (1999). Life course influences on African American men's depression: Adolescent parental composition, self-concept, and adult earnings. Journal of Black Studies, 29, 467-490. doi:10.1177/ 002193479902900401

Morgan, C., \& Cotton, S. R. (2003). The relationship between Internet activities and depressive symptoms in a sample of college freshmen.
Cyberpsychology \& Behavior, 6, 133-142. doi:10.1089/ 109493103321640329

Morgan, P. J., Lubans, D. R., Collins, C. E., Warren, J. M., \& Callister, R. (2009). Evaluation of the impact of an internet-based weight loss program for men: The SHED-IT randomized controlled trial. Obesity, 17, 2025-2032. doi:10.1038/oby.2009.85

Mulveen, R., \& Hepworth, J. (2006). An interpretive phenomenological analysis of participation in pro-anorexia internet site and its relationship with disordered eating. Journal of Health Psychology, 11, 283-296. doi:10.1177/1359105306061187

Oliver, W. (2006). "The streets": An alternative Black male socialization institution. Journal of Black Studies, 36, 918-937. doi:10.1177/ 0021934704273445

Parham, T. A., White, J. L., \& Ajamu, A. (2000). The psychology of Blacks: An African centered perspective. Upper Saddle River, NJ: Prentice Hall.

Paxton, K. C., Robinson, W. L., Shah, S., \& Schoeny, M. E. (2004). Psychological distress for African American adolescent males: Exposure to community violence and social support as factors. Child Psychiatry and Human Development, 34, 281-295. doi:10.1023/B CHUD.0000020680.67029.4f

Payne, Y. A. (2011). Site of resilience: A reconceptualization of resiliency and resilience in street life-oriented Black men. Journal of Black Psychology, 37, 426-451. doi:10.1177/0095798410394178

Payton, A. R. (2009). Mental health, mental illness, and psychological distress: Same continuum or distinct phenomena? Journal of Health and Social Behavior, 50, 210-227. doi:10.1177/002214650905000207

Petersen, J. L. (2009). "You have to be positive." Social support processes of an online social support group for men living with HIV. Communication Studies, 60, 526-541. doi:10.1080/10510970903260368

Pew Online Life Report. (2000). African-Americans and the Internet. Washington, DC: Pew Internet \& American Life Project. Retrieved from http://www.pewinternet.org

Pierre, M. R., \& Mahalik, J. R. (2005). Examining African self-consciousness and Black racial identity as predictors Black men's psychological wellbeing. Cultural Diversity and Ethnic Minority Psychology, 11, 28-40. doi:10.1037/1099-9809.11.1.28

Pierre, M. R., Mahalik, J. R., \& Woodland, M. (2001). The effects of racism, African self-consciousness and psychological functioning on Black masculinity: A historical and social adaptation framework. Journal of African American Men, 6, 19-39. doi:10.1007/s12111-0011006-2

Pieterse, A. L., \& Carter, R. T. (2007). An examination of the relationship between general life stress, racism-related stress, and psychological health among Black men. Journal of Counseling Psychology, 54, 101-109. doi 10.1037/0022-0167.54.1.101

Pinnock, C. B., \& Jones, C. (2003). Meeting the information needs of Australian men with prostate cancer by way of the Internet. Urology, 61, 1198-1203. doi:10.1016/SOD90-4295(03)00016-5

Plowden, K. O., John, W., Vasquez, E., \& Kimani, J. (2006). Reaching African American men: A qualitative analysis. Journal of Community Health Nursing, 23, 147-158.

Plowden, K., \& Young, A. E. (2003). Sociostructural factors influencing health behaviors of urban African-American men. Journal of National Black Nurses' Association, 14, 45-51.

Ravenell, J. E., Johnson, W. E., \& Whitaker, E. E. (2006). AfricanAmerica men's perceptions of health: A focus group study. Journal of the National Medical Association, 98, 544-550.

Rhodes, G., \& Lakey, B. (1999). Social support and psychological disorder: Insights from social psychology. In R. Kowalski (Ed.), The social psychology of emotional and behavioral problems: Interfaces of social and clinical psychology (pp. 281-309). Washington, DC: American Psychological Association. 
Rochlen, A. B., \& Hoyer, W. D. (2005). Marketing mental health to men. Journal of Clinical Psychology, 61, 675-684. doi:10.1002/jclp.20102

Rosenfarb, I. S., Bellack, A. S., \& Aziz, N. (2006). Family interactions and the course of schizophrenia in African American and White patients. Journal of Abnormal Psychology, 115, 112-120. doi:10.1037/0021843X.115.1.112

Rotondi, A. J., Hass, G. L., Anderson, C. M., Newhill, C. E., Spring, M. B., Ganguli, R., . . . Rosenstock, J. B. (2005). A clinical trial to test the feasibility of a telehealth pyschoeducational intervention for persons with schizophrenia and their families: Intervention and 3-month findings. Rehabilitation Psychology, 50, 325-336. doi:10.1037/0090-5550.50.4.325

Royster, M. O., Richmond, A., Eng, E., \& Lewis, M. (2006). Hey brother, how's your health? A focus group analysis of the health and healthrelated concerns of African American men in a southern city in the United States. Men \& Masculinities, 8, 389-404. doi10.1177/ 1097184X04268798

Scott, L. D., \& Davis, L. E. (2006). Young, Black, and male in foster care: Relationship of negative social contextual experiences to factors relevant to mental health service delivery. Journal of Adolescence, 29, 721-736. doi:10.1016/j.adolescence.2005.11.002

Sellers, S. L., Bonham, V., Neighbors, H. W., \& Amell, J. W. (2009). Effects of racial discrimination and health behaviors on mental and physical health of middle-class African American men. Health Education and Behavior, 36, 31-44. doi:10.1177/1090198106293526

Smedley, B. D., Stith, A. Y., \& Nelson, A. R. (Eds.). (2002). Unequal treatment: Confronting racial and ethnic disparities in health care. Washington, DC: National Academy Press.

Sullivan, C. F. (2003). Gendered cybersupport: A thematic analysis of two online cancer support groups. Journal of Health Psychology, 8, 83-104. doi: $10.1177 / 1359105303008001446$

U.S. Census Bureau. (2005). Income, earnings, and poverty data from the 2005 American Community Survey. Retrieved from http:// www.census.gov/prod/2006pubs/acs-02.pdf

Utsey, S. O. (1997). Racism and the psychological well-being of African American men. Journal of African American Men, 3, 69-87.

Wallington, S. F. (2008). The internet as an emerging patient education tool among African American men with prostate cancer: An exploratory study. American Journal of Mens Health, 2, 106-121. doi:10.1177/ 1557988306296156

Wang, H. H., Wu, S. Z., \& Liu, Y. Y. (2003). Association between social support and health outcomes: A meta-analysis. Kaohsiung Journal Medical Science, 19, 345-350. doi:10.1016/S1607-551X(09)70436-X

Wasserman, I. M., \& Richmond-Abbott, M. (2005). Gender and the Internet: Causes of variation in access, level, and scope of use. Social Science Quarterly, 86, 252-270. doi:10.1111/j.0038-4941.2005.00301.x

Watkins, D. C. (2011). Depression over the adult life course for African American men: Toward a framework for research and practice. American Journal of Men's Health. Advance online publication. doi:10.1177/ 1557988311424072

Watkins, D. C., Green, B. L., Goodson, P., Guidry, J., \& Stanley, C. A. (2007). Using focus groups to explore the stressful life events of Black college men. Journal of College Student Development, 48, 105-118.

Watkins, D. C., Green, B. L., Rivers, B. M., \& Rowell, K. L. (2006). Depression in Black men: Implications for future research. Journal of Men's Health and Gender, 3, 227-235. doi:10.1016/j/jmhg.2006.02.005
Watkins, D. C., Hudson, D. L., Caldwell, C. H., Siefert, K., \& Jackson, J. S. (2011). Discrimination, mastery, and depressive symptoms among African American men. Research on Social Work Practice, 21, 269-277. doi: 10.1177/1049731510385470

Watkins, D. C., \& Neighbors, H. W. (2007). An initial exploration of what 'mental health' means to young Black men. Journal of Men's Health and Gender, 4, 271-282.

Watkins, D. C., Walker, R. L., \& Griffith, D. M. (2010). A meta-study of Black male mental health and well-being. Journal of Black Psychology, 36, 303-330. doi:10.1177/0095798409353756

Wells, K., Klap, R., Koike, A., \& Sherbourne, C. (2001). Ethnic disparities in unmet needs for alcoholism, drug abuse, and mental health care. American Journal of Psychiatry, 158, 2027-2032.

Wester, S. R., Vogel, D. L., Wei, M., \& McLain, R. (2006). AfricanAmerican men, gender role conflict, and psychological distress: The role of racial identity. Journal of Counseling and Development, 84, 419429.

Weston, R. E., Weston, P. J., Futterman, R. F., Lepore, S. J., Carolina, D. S., Pinto, J. T., . . Gordon, A. P. (2007). Effectiveness of a modified computer assisted instructional tool in the dissemination of prostate cancer information to men of African descent through black churches Journal of African American Studies, 11, 140-156. doi:10.1007/s12111007-9017-2

Whaley, A. L. (2001). Cultural mistrust and mental health services for African Americans: A review and meta-analysis. Counseling Psychologist, 29, 513-531. doi:10.1177/0011000001294003

White, M., \& Dorman, S. M. (2001). Receiving social support online: Implications for health education. Health Education Research, 16, 693 707. doi:10.1093/her/16.6.693

Williams, D. R. (2003). The health of men: Structured inequalities and opportunities. American Journal of Public Health, 93, 724-731.

Williams, D. R., González, H. M., Neighbors, H., Nesse R., Abelson, J. M., Sweetman, J., \& Jackson, J. S. (2007). Prevalence and distribution of major depressive disorder in African Americans, Caribbean blacks, and non-Hispanic whites: Results from the National Survey of American Life. Archives of General Psychiatry, 64, 305-315. doi:10.1001/ archpsyc.64.3.305

Williams, D. R., \& Williams-Morris, R. (2000). Racism and mental health The African American experience. Ethnicity and Health, 5, 243-268.

Woodard, A. T., Taylor, R. J., Bullard, K. M., Neighbors, H. W., Chatters, L. M., \& Jackson, J. S. (2008). Use of professional and informal support by African Americans and Caribbean blacks with mental disorders. Psychiatric Services, 59, 1292-1298. doi:10.1176/appi.ps.59.11.1292

Woodard, A. T., Taylor, R. J., \& Chatters, L. M. (2011). Use of professional and informal support by Black men with mental disorders. Research on Social Work Practice, 21, 328-336. doi:10.1177/ 1049731510388668

Wright, K. (2000). Computer-mediated social support, older adults, and coping. Journal of Communication, 50, 100-118. doi:10.1111/j.14602466.2000.tb02855.x

Received March 15, 2011

Revision received January 5, 2012 Accepted January 10, 2012 